\title{
Severe obesity and gut microbiota: does bariatric surgery really reset the system?
}

\author{
Patrice D Cani
}

The gut microbiota is considered as one of the relevant contributors involved in the complex causes of obesity and related metabolic disorders. Indeed, a plethora of publications describing the composition of the microbiota in subjects suffering from overweight or obesity as well as type 2 diabetes and cardiometabolic diseases are available (for review ${ }^{1}{ }^{2}$ ). Conversely, the characteristics of the microbiome in extreme forms of obesity (ie, severe and morbid Body Mass Index $>35-40 \mathrm{~kg} / \mathrm{m}^{2}$ ) has been barely described.

Bariatric surgery has dramatically increased worldwide and is now appearing as a solution to reduce cardiovascular risks and diabetes, thereby leading to consider this surgical procedure as a 'metabolic surgery'. ${ }^{4}$ Interestingly, one of the key features of the microbiome characterising obesity is the so-called low microbial gene richness, which strongly correlates with metabolic disorders such as low-grade inflammation, insulin resistance, adipocyte size but also chance of success to respond to dietary intervention. ${ }^{5}$ However, we lack comprehensive data exploring the impact of bariatric surgery on severe obesity and the evolution of the microbiome (ie, composition, gene richness and activity) at several time points after the surgical intervention. Indeed, although some studies demonstrated changes in gut microbiota composition after bariatric

Correspondence to Professor Patrice D Cani, Université catholique de Louvain, WELBIO-Walloon Excellence in Life Sciences and BIOtechnology, Louvain Drug Research Institute, Metabolism and Nutrition Research Group, Brussels B-1200, Belgium; patrice. cani@uclouvain.be surgery, most of the investigations were performed using different techniques (eg, qPCR, 16S rDNA sequencing) in a small cohort of subjects and only very few of them used whole metagenome sequencing.

In GUT, Aron-Wisnewski and colleagues have recently filled this gap of knowledge by investigating in several cohorts of subjects the impact of bariatric surgeries (ie, adjustable gastric banding (AGB) and Roux-en-Y-gastric bypass (RYGB)) in severe obesity and at several time points (up to 12 months). ${ }^{7}$ Actually, in the last years, only very few studies have assessed the gut microbiota composition for up to 6 months after bariatric surgery. This paper is also probably the first in the field including humans with severe obesity and which deeply integrate numerous measurements such as metabolomics analysis but also high-resolution sequencing technology that has the advantage to access to known and unknown species abundance as well as precise functional profiling. It is worth noting that the authors have also extended their explorations in one of the cohorts and confirmed changes in the gut microbiome up to 5 years after bariatric surgery.

One of the interesting findings is that about $75 \%$ of the subjects with severe obesity exhibit a low microbial gene richness, which is far higher than in overweight or moderate obesity (ie, $40 \%$ of the subjects). ${ }^{5}$ As previously observed in other studies, the microbial gene richness was also associated with inflammation, insulin resistance and dyslipidaemia. ${ }^{5} 6$ However, in the present study, they also strongly linked the low microbial richness with trunk-fat and cardiometabolic complications (hypertension severity, glucose intolerance and type 2 diabetes). Furthermore, they found a specific signature of 78 metagenomic species characterising the status of low microbial richness. This means that 60 metagenomic species are novel as compared with the previous 18 identified during moderate obesity. ${ }^{8}$ Importantly, they not only found that most of them also correlates with metabolic parameters but they further confirmed this specific bacterial signature in another independent cohort composed of severely obese individuals who underwent RYGB.

Besides microbial signatures, the analysis of metabolites has led to the identification of nine serum metabolites (eg, glutarate, hippurate, 3-methoxyphenylacetic acid and L-histidine). Beyond specific correlations, the identification of these metabolites did not really point out to specific metabolic pathways or even mechanistic insight into the link between gut microbes, severe obesity and the onset of metabolic disorders. Therefore, further specific investigations are warranted to decipher if and how this kind of 'metabolomic signature' links severe obesity and related microbiota with any specific metabolic alterations.

As discussed earlier in this commentary, the researchers show that the gut microbiome is indeed correlated with several metabolic parameters. However, one of the most original findings is that the modulation of the gut microbiome after bariatric surgery did not completely follow the phenotype. In fact, despite a severe body weight loss and a strong improvement of several metabolic parameters, the subjects elected for a RYGB were still displaying a dramatic lower richness as compared with AGB 1 year after the bypass. More specifically, the bacterial species associated with a low bacterial richness (before surgery) were only marginally modified after the bariatric surgery and this 
despite numerous metabolic improvements. Therefore, this suggests that other mechanisms are responsible for the metabolic improvements observed after the surgical interventions. This observation also suggests that the low microbial richness cannot predict the presence, the absence or even the magnitude of the response to the RYGB procedure. Undeniably, this interesting finding strongly questions one of the current dogmas, which is currently installed in the field of bariatric surgery and microbiota, which is the fact that one part of the metabolic effects observed after a bariatric surgery (RYGB mostly) is due to specific changes in the gut microbiome.

As highlighted by the authors, one of the aims was to evaluate how severe obesity but also low microbial richness can be modified after an intervention and according to the type of surgery. Interestingly, they discovered that microbial gene richness increased 1 year after gastric bypass, but most of the subjects continued to exhibit a lower microbial gene richness and this despite significant improvement of their metabolism compared with gastric banding. It is also interesting to note that in a confirmation cohort, they also found an absence of complete microbial gene richness restoration even 5 years after the surgery. Therefore, this observation strongly highlights the fact that the reality is less simple than imagined.

In conclusion, this original study provides numerous data and elegantly integrates high-throughput data from independent cohorts. However, we are still at the beginning of a new era requiring further investigations. Indeed, it remains to demonstrate whether the low microbial gene richness as well as the metabolomics and species signatures are a cause or a consequence of the severe obesity. The dietary habits were not precisely investigated in the study; however, lifestyle factors (ie, dietary habits, activity, drugs) likely explain a large part of the microbiome composition variability. Thus, is this also the case in severe obesity? Are these signatures observed as a consequence of the duration or the history of obesity? Do they represent good biomarkers of gut microbiome alteration, eventually useful for patient stratification?

Twitter@MicrObesity

Contributors PDC wrote the commentary.

Funding PDC is a senior research associate at FRS-FNRS (Fonds de la Recherche Scientifique), recipient of grants from FNRS (FRFS-WELBIOWELBIO-CR-2017C-02), The Excellence Of Science (EOS-30770923), the Funds Baillet Latour (Grant for Medical Research 2015) and ERC Starting Grant 2013 (336452-ENIGMO).

Competing interests $\mathrm{PDC}$ is inventor on patent applications dealing with the use of $A$. muciniphila and its components in the treatment of obesity and related disorders. PDC is co-founder of A-Mansia Biotech SA.

Provenance and peer review Commissioned; internally peer reviewed.

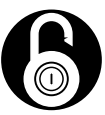

\section{OPEN ACCESS}

Open access This is an open access article distributed in accordance with the Creative Commons Attribution Non Commercial (CC BY-NC 4.0) license, which permits others to distribute, remix, adapt, build upon this work non-commercially, and license their derivative works on different terms, provided the original work is properly cited, appropriate credit is given, any changes made indicated, and the use is non-commercial. See: http:// creativecommons.org/licenses/by-nc/4.0/.

(c) Author(s) (or their employer(s)) 2019. Re-use permitted under CC BY-NC. No commercial re-use. See rights and permissions. Published by BMJ.

\section{Check for updates}

To cite Cani PD. Gut 2019;68:5-6.

Received 7 June 2018

Revised 25 June 2018

Accepted 27 June 2018

Published Online First 10 July 2018

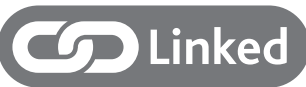

http://dx.doi.org/10.1136/gutjnl-2018-316103

Gut 2019;68:5-6.

doi:10.1136/gutjnl-2018-316815

\section{REFERENCES}

1 Shanahan F, van Sinderen D, O'Toole PW, et al. Feeding the microbiota: transducer of nutrient signals for the host. Gut 2017:66:1709-17.

2 Cani PD, Jordan BF. Gut microbiota-mediated inflammation in obesity: a link with gastrointestinal cancer. Nat Rev Gastroenterol Hepatol 2018 [Epub ahead of print 29 May 2018].

3 Bedossa P, Tordjman J, Aron-Wisnewsky J, et al. Systematic review of bariatric surgery liver biopsies clarifies the natural history of liver disease in patients with severe obesity. Gut 2017;66:1688-96.

4 Panunzi S, Carlsson L, De Gaetano A, et al. Determinants of diabetes remission and glycemic control after bariatric surgery. Diabetes Care 2016;39:166-74.

5 Cotillard A, Kennedy SP, Kong LC, et al. Dietary intervention impact on gut microbial gene richness. Nature 2013:500:585-8.

6 Dao MC, Everard A, Aron-Wisnewsky J, et al. Akkermansia muciniphila and improved metabolic health during a dietary intervention in obesity: relationship with gut microbiome richness and ecology. Gut 2016:65:426-36.

7 Aron-Wisnewsky J, Prifti E, Belda E, et al. Major microbiota dysbiosis in severe obesity: fate after bariatric surgery. Gut 2018;68:70-82.

8 Le Chatelier E, Nielsen T, Qin J, et al. Richness of human gut microbiome correlates with metabolic markers. Nature 2013:500:541-6. 